\title{
REFLEXIVE LATTICES OF SUBSPACES ${ }^{1}$
}

\author{
K. H. KIM AND F. W. ROUSH
}

\begin{abstract}
A lattice of subspaces of a vector space is called reflexive if no other subspace is invariant under all linear mappings which leave invariant all subspaces of the lattice. We characterize finite reflexive lattices of subspaces of a finite dimensional vector space over an infinite field.
\end{abstract}

The subspaces of a vector space form a lattice under the operations $\Lambda=\cap, \vee=$ span of two subspaces. Any sublattice of this lattice will be called a lattice of subspaces.

Definition 1. A lattice $L$ of subspaces of a vector space is reflexive if whenever $W \notin L$ there exists a linear transformation $A$ such that $A W \not \subset W$ but $A V \subset V$ for all $V \in L$.

A lattice of subspaces of a vector space will be reflexive if and only if it is lattice of submodules of an algebra $A^{\prime} \subset A=$ End $V$.

P. R. Halmos ([2], [3]) has proposed the problem of characterizing reflexive lattices of subspaces. In this paper we solve this problem for finite lattices of subspaces of a finite dimensional vector space, over an infinite field.

Definition 2. A lattice is distributive if and only if the identities $(x \wedge y) \vee$ $z=(x \vee z) \wedge(y \vee z)$ and $(x \vee y) \wedge z=(x \wedge z) \vee(y \wedge z)$ are always valid.

A lattice is distributive if and only if it is isomorphic to a lattice of sets.

LEMMA 1. Let $L$ be a lattice of subsets of $N=\{1,2, \ldots, n\}$ containing $\varnothing$ and $N$. Let $W$ be a set not belonging to $L$. Then there exists a transformation $f$ such that $f(S) \subset S$ for $S \in L$ but $f(W) \not \subset W$.

Proof. The lattice $L$ gives a topology on $N$, if we consider the members of $L$ to be the closed sets of the topology. Since $W$ is not in $L$, it is not closed. Therefore there exists $u \in W$ such that $\bar{u}$ is not contained in $W$. Let $v \in \bar{u} \backslash W$. Now define $f$ by $f(x)=x, x \neq u$ and $f(u)=v$. Since $f(u)=v$, $f(W) \not \subset W$. Let $S \in L$. If $u \notin S$, then $f(S)=S$ since $f$ will be the identity on $S$. If $u \in S$ then $f(S) \subset S \cup f\{f(u)\}=S \cup\{v\}$. Since $S$ is closed, $\bar{u} \subset S$. Therefore $v \in S$. So $S \cup\{v\}=S$. Therefore $f(S) \subset S$. This completes the proof.

Received by the editors September 15, 1978 and, in revised form, February 28, 1979. AMS (MOS) subject classifications (1970). Primary 15A03; Secondary 06A20, 46C10.

Key words and phrases. Lattices of subspaces, distributive lattice, invariant subspaces, transformations, infinite field.

${ }^{1}$ This work was supported by Alabama State University Faculty Research Grant R-78-6. 
THEOREM 2. Let $V$ be a finite dimensional vector space over an infinite field. Let $L$ be a finite lattice of subspace of $V$. Then $L$ is reflexive if and only if it is distributive.

Proof. Suppose $L$ is distributive. Let $\left\{C_{i}\right\}$ be a lattice basis for $L$, i.e. a minimal set of members of $L$ which together with $\{0\}$ generate the lattice under join. Every finite lattice has a unique basis, namely the join-irreducible elements.

For a basis element $C_{i}$, let $\hat{C}_{i}$ be the span of all members of $L$ which are proper subspaces of $C_{i}$. Then $\hat{C}_{i}$ is a subspace of $C_{i}$. Choose subspaces $V_{i}$ such that $C_{i}=\hat{C}_{i} \oplus V_{i}$. Let $w \in V_{k} \cap \Sigma_{i \neq k} V_{i}$. Then

$$
w \in C_{k} \cap \sum_{i \neq k} C_{i}=\sum C_{i} \cap C_{k} \subset \hat{C}_{k} .
$$

Since $w \in V_{k} \cap \hat{C}_{k}, w=0$. This proves that $\Sigma V_{i}$ is a direct sum.

We next assert that each subspace $C_{i}$ is the direct sum of those $V_{k}$ contained in $C_{i}$. Suppose not. Let $C_{j}$ be a minimal member of $\left\{C_{i}\right\}$ which is not spanned by subspaces $V_{k} \subset C_{j}$. Then $\hat{C}_{j}$ is a join of proper subspaces $C_{s} \subset C_{j}$ so $\hat{C}_{j}$ is spanned by $V_{i}: V_{t} \subset \hat{C}_{j}$. But $C_{j}=\hat{C}_{j} \oplus V_{j}$. This is a contradiction, and proves the assertion.

Now let $Y$ be a subspace of $V$ which does not belong to $L$. To show that $L$ is reflexive, it will suffice to show that there exists a linear mapping $V \rightarrow V$ under which each member of $L$ is invariant, but under which $Y$ is not invariant.

If $Y$ is a direct sum of subspaces $V_{t}$, then the existence of such a linear mapping follows from Lemma 1 . Let $\pi_{i}$ denote the projection $V=\bigoplus V_{j} \rightarrow$ $V_{i}$. If for some $i, \pi_{i}(Y) \not \subset Y$ let $\pi_{i}$ be the mapping. So assume $\pi_{i}(Y) \subset Y$ for each $i$. If $0 \neq \pi_{i}(Y) \neq V_{i}$ for some $i$, choose a linear mapping which is the identity on all $V_{j}, j \neq i$, and which sends $V_{i}$ into $V_{i}$ but does not preserve $\pi_{i}(Y)$. So we may assume $\pi_{i}(Y)=V_{i}$ or $\pi_{i}(Y)=0$ for each $i$, and $\pi_{i}(Y) \subset Y$. Thus if $y \in Y$ has a nontrivial component in $V_{i}$, then all of $V_{i}$ lies in $Y$. So $Y$ is a direct sum of subspaces $V_{t}$, and we are in a case previously dealt with. This proves that a finite distributive lattice of subspaces is reflexive. The converse follows from [4, Lemma 1.2].

ACKNOWLEDGEMENT. The authors would like to thank the referee for a very helpful suggestion on the original draft of this paper.

\section{REFERENCES}

1. G. Birkhoff, Lattice theory, Amer. Math. Soc. Colloq. Publ., no. 25, Amer. Math. Soc., Providence, R. I., 1967.

2. P. R. Halmos, Finite dimensional Hilbert spaces, Amer. Math. Monthly 77 (1970), 457-464.

3. __ Reflexive lattices of subspaces, J. London Math. Soc. (2) 4 (1971), 257-263.

4. J. P. Jans, On the indecomposable representations of algebras, Ann. of Math. (2) 66 (1957), 418-429.

Mathematics Research Group, Alabama State University, Montgomery, Alabama 36101 